\title{
Integrin-targeted AmpRGD sunitinib liposomes as integrated antiangiogenic tools
}

\author{
Francesca Bianchini, $\mathrm{PhD}^{\mathrm{a}, 1}$, Augusta De Santis, $\mathrm{PhD}^{\mathrm{b}, 1}$, Elisabetta Portioli, $\mathrm{PhD}^{\mathrm{c}}$, \\ Irene Russo Krauss, $\mathrm{PhD}^{\mathrm{b}}$, Lucia Battistini, $\mathrm{PhD}^{\mathrm{c}}$, Claudio Curti, $\mathrm{MS}^{\mathrm{c}}$, Silvia Peppicelli, $\mathrm{PhD}^{\mathrm{a}}$, \\ Lido Calorini, $\mathrm{MD}, \mathrm{PhD}^{\mathrm{a}}$, Gerardino D'Errico, $\mathrm{PhD}^{\mathrm{b}}$, Franca Zanardi, $\mathrm{PhD}^{\mathrm{c}, *}$, \\ Andrea Sartori, $\mathrm{PhD}^{\mathrm{c}, *}$ \\ ${ }^{a}$ Dipartimento di Scienze Biomediche, Sperimentali e Cliniche "Mario Serio", Università degli Studi di Firenze, Firenze, Italy \\ 'Dipartimento di Scienze Chimiche, Università degli Studi di Napoli "Federico II", Napoli, Italy \\ ${ }^{\mathrm{c}}$ Dipartimento di Scienze degli Alimenti e del Farmaco, Università di Parma, Parma, Italy
}

Revised 8 February 2019

\begin{abstract}
We report here the preparation, physico-chemical characterization, and biological evaluation of a new liposome formulation as a tool for tumor angiogenesis inhibition. Liposomes are loaded with sunitinib, a tyrosine kinase inhibitor, and decorated with cyclo-aminoprolineRGD units (cAmpRGD), efficient and selective ligands for integrin $\alpha_{V} \beta_{3}$. The RGD units play multiple roles since they target the nanovehicles at the integrin $\alpha_{V} \beta_{3}$-overexpressing cells (e.g. activated endothelial cells), favor their active cell internalization, providing drug accumulation in the cytoplasm, and likely take part in the angiogenesis inhibition by interfering in the $\alpha_{\mathrm{V}} \beta_{3}$-VEGFR2 cross-talk. Both in vitro and in vivo studies show a better efficacy of this integrated antiangiogenic tool with respect to the free sunitinib and untargeted sunitinib-loaded liposomes. This system could allow a lower administration of the drug and, by increasing the vector specificity, reduce side-effects in a prolonged antiangiogenic therapy.
\end{abstract}

(C) 2019 Elsevier Inc. All rights reserved.

Key words: Liposomes; Integrin $\alpha_{\mathrm{V}} \beta_{3}$; RGD; Tumor angiogenesis; Sunitinib

\section{Introduction}

Tumor angiogenesis is the formation of new blood vessels from pre-existing vasculature, and it is triggered by several pro- angiogenic factors, when a growing tumor needs the supply of oxygen and nutrients. ${ }^{1}$ Several years ago, inhibition of tumor angiogenesis was recognized as an alternative strategy to hinder the tumor growth with the idea to starve cancer cells, and many

Abbreviations: cAmpRGD, cyclic 4-aminoproline-RGD; EE, encapsulation efficiency; VEGF, vascular endothelium growing factor; PDGFR, plateletderived growth factor receptor; DSPE-PEG 2000 -DBCO, 1,2-distearoyl-sn-glycero-3-phosphoethanolamine- $N$-[dibenzocyclooctyl(polyethylene glycol)-2000] (ammonium salt); POPC, 1-palmitoyl-2-oleoyl-sn-glycero-3-phosphocholine; CHOL, cholesterol; n-PCSL, $\mathrm{n}=5$ or 14 1-palmitoyl-2[n-(4,4-dimethyloxazolidine- $N$-oxyl)]-stearoyl-sn-glycero-3-phosphocholine; DLS, dynamic light scattering; ELS, electrophoretic light scattering; cryo-TEM, cryogenic transmission electron microscopy; FITC, fluorescein isothiocyanate; PBS, phosphate buffer solution.

Acknowledgments: The authors thank the Centro Interdipartimentale Misure "G. Casnati" (University of Parma) for instrumental facilities. This work was supported by the Italian Ministry of Education, University and Research (MIUR) (PRIN 2015 contract no. 20157WW5EH). This work was also supported by Ente Cassa di Risparmio di Firenze and the Istituto Toscano Tumori (Calorini 2013).

Statement of conflict of interest: No conflict of interest exists in the submission of this manuscript, and manuscript is approved by all authors for publication.

*Corresponding authors.

E-mail addresses: franca.zanardi@unipr.it, (F. Zanardi), andrea.sartori@unipr.it. (A. Sartori).

1 These authors contributed equally 
antiangiogenic drugs were approved for cancer treatment, most of them being directed toward the VEGFs and their receptors (VEGFRs). ${ }^{2,3}$ In fact, the couple VEGF-A-VEGFR2, in particular, was recognized as the main actor in tumor angiogenesis. ${ }^{4}$ The approved drugs are of different nature and include monoclonal antibodies, recombinant proteins and tyrosine kinase inhibitors (TKIs). Sunitinib, for example, is a multi-targeted TKI, a small molecule able to hamper in particular the phosphorylation of VEGFR2 and PDGFR, and it has been approved as single-treatment agent for metastatic renal cell carcinoma and pancreatic neuroendocrine tumors. ${ }^{5}$ Despite the promises in the clinical phases, the antiangiogenic treatments have provided only limited benefits. The reasons are several and include: i) the lack of specific biomarkers making impossible any selection of patients who could likely benefit from antiangiogenic drugs; ii) the fact that most of the approved antiangiogenic drugs are used for the treatment of advanced-stage disease, and iii) the onset of resistance to the therapy by upregulation of other angiogenic factors or compensatory pathways. ${ }^{6-8}$ Moreover, the excessive blood vessel pruning causes hypoxia and can promote cancer metastasis. For these reasons, recent approaches in the optimization of anti-angiogenic treatments are aimed at tumor vascular normalization, by a properly timed low dosage of antiVEGF or anti-VEGFR therapeutics. ${ }^{9-11}$ One of the trends showing clinical benefits is a long-term anti-angiogenesis therapy that can be realized only with drugs that have relatively low toxicity and high tolerability, ${ }^{9}$ while possibly being able to hit several pathways involved in angiogenesis at one time.

Integrins are another class of receptors that play an active role in cancer progression and tumor angiogenesis. ${ }^{12-14}$ Integrin $\alpha_{V} \beta_{3}$, in particular, recognizes the tripeptide sequence RGD present in the extracellular matrix proteins, is overexpressed on many solid tumors and activated endothelial cells during angiogenesis and has high expression on tumor vasculature, ${ }^{15}$ potentiating the activity of tyrosine kinase receptors. ${ }^{16} \mathrm{~A}$ widely attested cross-talk between $\alpha_{V} \beta_{3}$ and VEGFR2 enhances the activity of both receptors and increases the VEGF-induced angiogenesis. ${ }^{17-19}$ For these reasons, integrin $\alpha_{V} \beta_{3}$ has been considered for years both a good tumor marker useful to target RGD-decorated drugs or nanoparticles (NPs), ${ }^{20,21}$ and a therapeutic target for antiangiogenic therapy ${ }^{15,22}$; however, the recent failure of the clinical phase III of cilengitide - a potent RGD-based ligand of integrin $\alpha_{V} \beta_{3}$ - in the cure of glioblastoma, ${ }^{23}$ lowered the interest in this integrin subclass as a therapeutic target.

The controversial clinical results of cilengitide and other integrin-directed agents highlighted the complex scenario of the actual role of integrins in cancer, due to individual variability of their expression, activity, availability and activation states together with the difficulty to find the right timing and dosage in administration of an integrin inhibitor. ${ }^{13,24,25}$ Nevertheless, according to several researchers, these difficulties should not hamper the development of new therapeutic approaches or drugs to target cancer-related integrins that possibly modulate multiple yet intertwined angiogenic pathways. ${ }^{25}$

Our research group developed high-affinity $\alpha_{\mathrm{V}} \beta_{3}$-ligands, namely cyclic 4-aminoproline-RGD peptidomimetics (cAmpRGD), that showed remarkable and selective binding capability in both cell-free and cell assays. ${ }^{26,27}$ Recently, these cAmpRGD ligands were covalently joined to a sunitinib-like portion, to furnish dual compounds aimed at targeting the $\alpha_{\mathrm{V}} \beta_{3}$ integrin/VEGFR2 receptor couple, with superior antiangiogenesis properties as compared to the single modules (cAmpRGD and sunitinib) or their simple combination. ${ }^{28}$ The cAmpRGD units were also used to decorate liposomes loaded with doxorubicine to direct the NPs at the cancer cells overexpressing the $\alpha_{V} \beta_{3}$ integrin thus increasing the liposome uptake. ${ }^{29}$

On the basis of these results, our goal was to develop novel targeted cAmpRGD-liposomes loaded with sunitinib, to produce a system capable to exploit both the passive and the active targeting at the tumor site. Even if a liposome can extravasate and accumulate in the tumor site because of fenestrated tumor blood vessels and poor lymphatic drainage (enhanced permeation and retention effect), the active targeting can allow the liposome nanoparticles to overcome the high tumor interstitial fluid pressure and reach the inner tumor layers. ${ }^{30}$ The cAmpRGD ligands should also increase the NP internalization via an integrin-mediated endocytosis, ${ }^{31}$ thus improving the therapeutic index of sunitinib, and cooperate with sunitinib in the angiogenesis inhibition. Such a system could likely allow a spatio-temporal co-delivery of the two active units, and a lower sunitinib administration for a more effective and tolerable prolonged therapy.

\section{Methods}

Details on materials, instrumentations, methods and experimental procedures are available in the Supporting Information.

Synthesis of c(AmpRGD)-N $\mathbf{N}_{3} \mathbf{2}$ and DSPE-PEG-RGD 3 (Figure 2) are reported in SI.

\section{Liposome preparation and characterization}

Different liposomes were prepared (see Fig. 1), namely: (a) LN, consisting of POPC/CHOL/DSPE-PEG $2000-\mathrm{DBCO}$ (55/40/ $5 \mathrm{~mol} / \mathrm{mol}$ ); (b) RGD-LN, consisting of POPC/CHOL/DSPEPEG-RGD (55/40/5 mol/mol); (c) PCSL-LN, consisting of POPC/CHOL/n-PCSL/DSPE-PEG-RGD (54:40:1:5 mol/mol) for electron paramagnetic resonance (EPR) studies; d) nonstealth liposomes noS-LN, consisting of POPC/CHOL (60/ $40 \mathrm{~mol} / \mathrm{mol}$ ). All the liposomes were prepared by the thin film layer method, ${ }^{29}$ and characterized by DLS ${ }^{32}$ and ELS measurements. Cryo-TEM images of RGD-LNs were taken.

\section{Sunitinib loading}

The sunitinib encapsulation was performed by exploiting an ammonium sulfate gradient ${ }^{33}$ to obtain a $20 \%$ by mol with respect to the lipid content. The encapsulation efficiency (EE), defined as the percentage of drug loaded into liposomes relative to the total amount of drug, was evaluated after ultracentrifugation procedures, through UV-Vis measurements.

\section{Sunitinib leakage}

The liposomal release of sunitinib was quantified by the fluorescence associated to sunitinib malate upon dialysis of the 


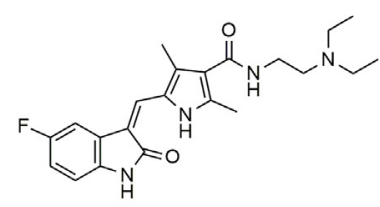

Sunitinib $=$

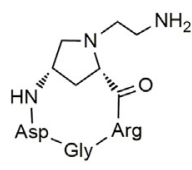

cAmpRGD-NH ${ }_{2}$

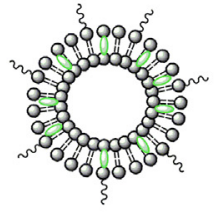

LN

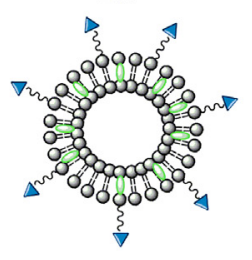

RGD-LN
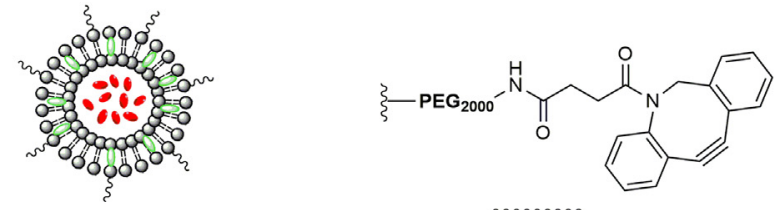

LN-(sun)

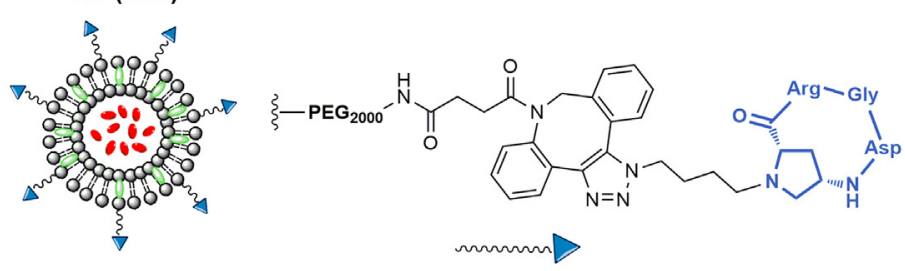

RGD-LN-(sun)

Figure 1. Compound structures and schematic representation of liposome formulations used in this work.

liposomes. ${ }^{34,35}$ PBS with $10 \%$ Dulbecco's modified eagle's medium was chosen as release medium. The change of fluorescence intensity of the medium was monitored at different time intervals (details in SI).

\section{Cell lines}

Endothelial progenitor cells (EPCs) were from human umbilical cord blood samples of healthy newborns as previously described. $^{36}$

\section{Inhibition of EPC adhesion to the $\alpha_{V} \beta_{3}$-ligand vitronectin}

Suspended EPCs were treated with increasing concentrations of the ligands, before plating them on vitronectin-coating wells. After incubation, adherent cells were counted and the inhibitory activity was calculated as percentage of cell adhesion to vitronectin compared to untreated.

\section{Inhibition of EPC growth}

EPCs, seeded on gelatin-coated plates, were exposed to a serum-free medium supplemented with $20 \mathrm{ng} / \mathrm{mL}$ VEGFA containing sunitinib $1 \mu \mathrm{M}$ as a free drug or in liposome formulations, or the corresponding concentration of blank liposomes. The number of the vital cells was determined at $24 \mathrm{~h}, 48 \mathrm{~h}$, and $72 \mathrm{~h}$.

\section{Cellular uptake of sunitinib}

Internalization of sunitinib was determined by flow cytometer using the FITC channel, taking advantage of the fluorescent emission of sunitinib.

\section{Inhibition of VEGFR2 phosphorylation}

Serum-deprived EPCs were pre-treated with sunitinib $1 \mu \mathrm{M}$ as a free drug or in liposome formulation for $24 \mathrm{~h}$ and then stimulated with VEGFA $(50 \mathrm{ng} / \mathrm{mL})$ for $3 \mathrm{~min}$. Cells were then lysed and phosphorylated VEGFR2 on tyr951 detected.

\section{Inhibition of in vitro and in vivo angiogenesis}

The effects of the different liposome formulations on the inhibition of the capacity of EPCs to differentiate into capillarylike network were in vitro assessed by Matrigel morphogenesis assay, while the in vivo efficacy of these compounds was evaluated by Matrigel sponge assay on subcutaneously implanted FVB mice.

\section{Statistical analysis}

Data are expressed as the mean \pm standard deviation (SD). When appropriate, results are shown as normalized data. Data were analyzed using analysis of variance followed by a multiplecomparison Student-Newman-Keuls test to identify means that are different from each other. Mean values are considered significantly different when $P<0.05$, and are indicated with unlike letters (a, b, c, d, e, f).

All experimental procedures involving animals were performed in accordance with national guidelines, approved by the ethical committee of Animal Welfare Office of Italian Work Ministry (401/2015PR approved 05/21/2015) and conformed to the legal mandates and Italian guidelines for the care and maintenance of laboratory animals.

\section{Results}

\section{Synthesis of cAmpRGD-N 2 and phospholipid-RGD $\mathbf{3}$}

The integrin ligand $\mathbf{2}$ was obtained by acidic deprotection of the corresponding precursor, whose synthesis was previously described $^{28}$ (Scheme S1). Compound 2 was linked to the DSPEPEG(2000)-DBCO via an azide-alkyne Huisgen cycloaddition reaction (click chemistry) (Figure 2). The use of the dibenzoazacyclooctyne, in which the alkyne group is inserted in a highly tensioned cycle, allows the reaction to take place very rapidly and without the use of a $\mathrm{Cu}(\mathrm{I})$ catalytic activation or any other salt, making the purification of the delicate phospholipid-RGD adduct 3 very simple. The reaction was almost quantitative and the product formation was confirmed by electrospray mass 


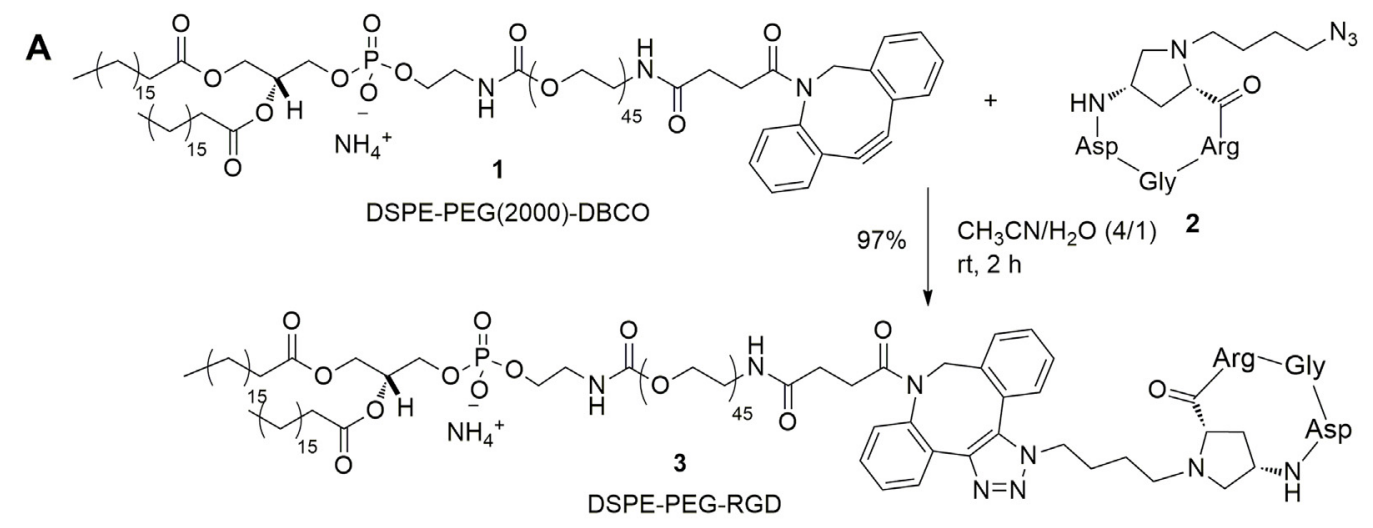

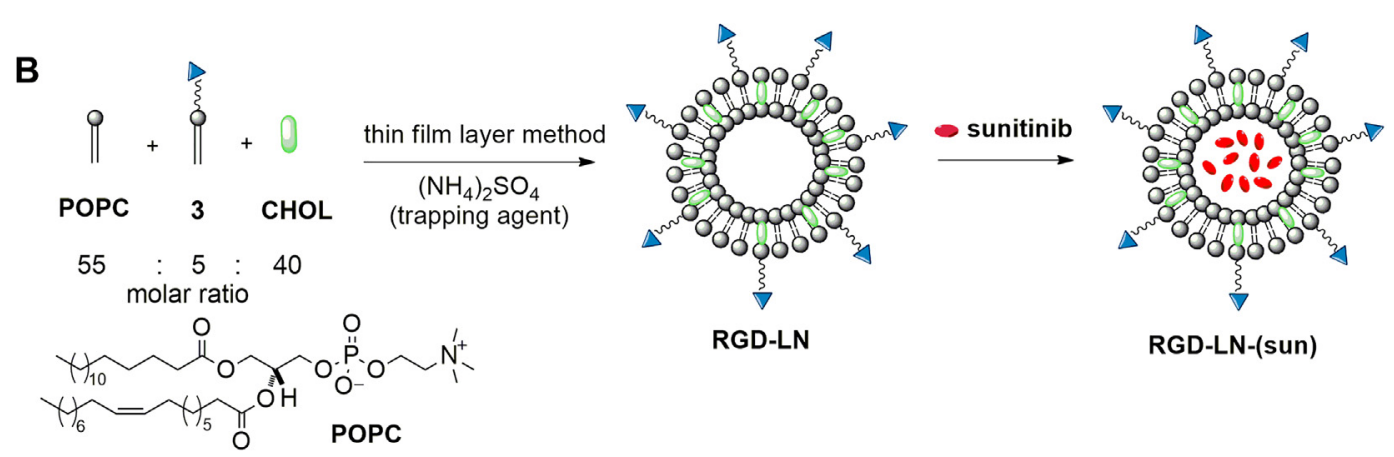

Figure 2. (A) Synthesis of the phospholipid DSPE-PEG-RGD 3; (B) liposome preparation and sunitinib encapsulation.

Table 1

Liposome characterization.

\begin{tabular}{llll}
\hline Liposome & $\begin{array}{l}\text { Particle size } \\
(\mathrm{nm})\end{array}$ & Polydispersity index & $\begin{array}{l}\text { Zeta potential } \\
(\mathrm{mV})\end{array}$ \\
\hline LN & $69 \pm 4$ & $0.13 \pm 0.04$ & $-5.5 \pm 0.5$ \\
LN-(sun) & $65 \pm 3$ & $0.16 \pm 0.06$ & $-4.7 \pm 0.6$ \\
RGD-LN & $73 \pm 2$ & $0.21 \pm 0.01$ & $-5.3 \pm 0.7$ \\
RGD-LN-(sun) & $66 \pm 3$ & $0.25 \pm 0.05$ & $-4.5 \pm 0.5$ \\
\hline
\end{tabular}

analysis. The phospholipid-RGD 3 was used in the preparation of targeted liposomes RGD-LNs in a $5 \mathrm{~mol} \%$ with respect to the other constituents of the lipid bilayer.

\section{Liposome preparation and characterization}

The phospholipid-RGD 3 was co-formulated with commercial lipids (POPC and CHOL) to obtain the targeted nano-vehicles for sunitinib. The physicochemical investigation of the integrin-directed liposomal nanoparticles was performed using DLS to estimate liposome dimensions, ELS for the zeta-potential calculation and EPR to investigate the dynamics of the lipid hydrophobic tail in the bilayer. ${ }^{37}$ The mean hydrodynamic radii $\left\langle\mathrm{R}_{\mathrm{H}}\right\rangle$ for the aqueous dispersions of LN, LN-(sun), RGD-LN and RGD-LN-(sun) liposome-based systems (Figure S2 and Table 1) are in the 65$75 \mathrm{~nm}$ range, which is the typical range of large unilamellar vesicles. Furthermore, a moderate polydispersity was observed for all the aggregates, with $\langle\mathrm{ID}\rangle$ values ranging between 0.13 and 0.25 . Membrane unilamellarity and spherical shape of these liposomes were also confirmed by the cryo-TEM images (Figure S3). The surface of the blank liposomes is slightly negative (zeta-potential $-5.4 \mathrm{mV}$ ), and the sunitinib loading produces only a slight change on the surface charge density. The EPR spectroscopy was utilized to obtain information on the acyl chains structuring the lipid bilayers. ${ }^{38}$ EPR experiments were performed employing the spin-label approach, analyzing spectra of phosphocholines spin-labeled on the C5 or C14 atom of the sn-2 chain (5-PCSL and 14-PCSL, respectively) incorporated in non-stealth liposomes (noS-LNs) and RGD-LN liposomes. 5-PCSL bears the radical label close to the molecule headgroup and allows the behavior of the region of the membrane closer to the polar external layers to be monitored. In contrast, 14-PCSL bears the radical label close to the terminal methyl group of the acyl chain, thus allowing the monitoring of the deep hydrophobic core of the bilayer. In all the systems, the 5-PCSL and 14-PCSL spectra present anisotropic line-shapes (Figure S4), indicating that the lipids are organized in a lyotropic liquid crystalline phase more ordered than the one observed for pure POPC. ${ }^{39}$ Effects of phospholipid-RGD 3 are weakly detectable, indicating scarce perturbation of the bilayer structuring. This was quantitatively confirmed by the determination of the spin-label isotropic hyperfine coupling constant, $a_{N}$, and the order parameter, $S$, which are the index of the micropolarity experienced by the nitroxide and the index of the motion of the acyl chain segment to which the label is bound, respectively (details in SI, Table S1). ${ }^{40}$

\section{Sunitinib loading in lipid nanoparticles}

A modified ammonium salt gradient method ${ }^{33}$ was successfully performed to load sunitinib into liposomes, and the encapsulation was verified by $\mathrm{UV}-\mathrm{V}$ is spectroscopy. In the 

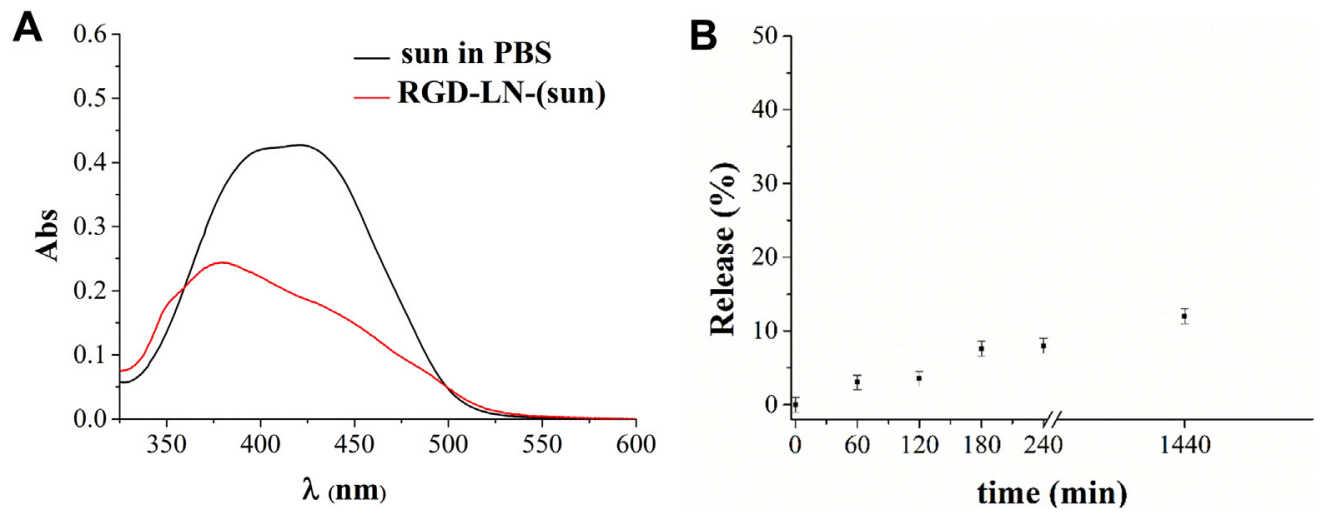

Figure 3. (A) UV-Vis spectra of $200 \mu \mathrm{M}$ solutions of sunitinib in PBS (black) and in RGD-LN (red). (B) Sunitinib release, expressed as \%, quantified through fluorescence upon dialysis.

range of interest, calibration curves for sunitinib malate were determined both in PBS and $\left(\mathrm{NH}_{4}\right)_{2} \mathrm{SO}_{4}$ (see SI). Notably, sunitinib UV-Vis spectra in ammonium salt solution show two hallmarks: i) a blue shift of the absorbance peak; ii) a shoulder at $350 \mathrm{~nm}$ (Figure S5), which strongly differ from spectral features in water and provide a reliable method to assess encapsulation of the drug within the liposome. The UV-Vis spectrum of sunitinib in the presence of liposomes with $\left(\mathrm{NH}_{4}\right)_{2} \mathrm{SO}_{4}$ in the inner aqueous pool (Figure 3,A) shows both these two features confirming the encapsulation of sunitinib in the liposomes interior.

To estimate the encapsulation efficiency, a liposome sample was centrifuged and the sunitinib concentration in the supernatant was determined by UV-Vis. Knowing the amount of sunitinib used for encapsulation experiments, an EE\% $>96 \%$ w/ w was determined. Pellet deriving from this experiment was resuspended and stored at $4{ }^{\circ} \mathrm{C}$ in order to check the stability of the formulation over time. No change in the liposome size distribution (monitored by DLS) and in the UV spectrum was observed up to two weeks.

\section{Sunitinib leakage from lipid nanoparticles}

To investigate the ability of lipid nanoparticles to maintain sunitinib in their interior for a prolonged time, the RGD-LNs(sun) were put in a dialysis tube and dialyzed against DME medium, a medium with higher viscosity than PBS that can well mime diffusion events of sunitinib in biological assays. The percentage of sunitinib released through diffusion mechanism over the first 24 hours is less than $12 \%$ (Figure 3, B).

\section{Effect of RGD-liposome formulations on EPC adhesion and viability}

To evaluate the ability of the targeted liposomes (RGD-LNs) to interact with $\alpha_{V} \beta_{3}$ integrins on the surface of endothelial cells, an inhibition assay of adhesion of EPCs to vitronectin was performed. The expression of $\alpha_{V} \beta_{3}$ integrins on EPCs was assessed by cytofluorimetry to be $>87 \%$ (while the expression of $\alpha_{\mathrm{V}} \beta_{5}$ and $\alpha_{5} \beta_{1}$, other RGD-recognizing integrins, resulted negligible, Figure S7). Blank liposomes (LNs) and the ligand cAmpRGD-NH $\mathrm{N}_{2}$ were also used. The assay was performed with increasing concentrations of compounds in the range $0.5-5000 \mathrm{nM}$. In case of RGD-LNs, the concentration refers to that of the RGD units on the outer layer, while in case of untargeted LNs it was used the same liposomal concentration of the corresponding RGD-LNs.

The targeted RGD-LNs are able to strongly inhibit the EPC adhesion to vitronectin even at $0.5 \mathrm{nM}$, at which concentration the inhibition is $\sim 50 \%$ (Figure $4, A$ ). The comparison with the inhibition provided by the cAmpRGD-NH $\mathrm{N}_{2}$ shows a clear multivalent effect, given by the multimeric cAmpRGD presentation on the liposomes. With cAmpRGD- $\mathrm{NH}_{2}, 50 \%$ of inhibition is reached at $1 \mu \mathrm{M}$ ligand concentration, three orders of magnitude higher than RGD-LNs. On the contrary, the untargeted liposomes are not able to inhibit EPCs adhesion at any concentration.

The inhibition of EPCs growth instead was evaluated after $24 \mathrm{~h}$, $48 \mathrm{~h}$ and $72 \mathrm{~h}$ incubation with the diverse liposomal formulations or the free drug at $1 \mu \mathrm{M}$ concentration, referred to sunitinib as free drug or encapsulated in the liposome. Blank liposomes were used at the same concentration of the loaded liposomes. Both LNs and RGD-LNs are not cytotoxic and do not inhibit the cell growth, confirming the biocompatibility of the targeted RGD-LNs as nanovehicles. The loaded targeted liposomes RGD-LN-(sun) showed an inhibitory effect comparable to that of free sunitinib at $24 \mathrm{~h}$, but stronger at $48 \mathrm{~h}$ and $72 \mathrm{~h}$. Moreover, the activity of RGD-LN-(sun) is superior to that of LN-(sun), and this is likely due to an active role of cAmpRGD units in the internalization of the nanoparticles. In fact, since the corresponding blank formulation RGD-LN has no influence on the cell growth, the stronger inhibition by RGD-LN-(sun) can be explained only with a higher intracellular concentration of sunitinib (Figure 4, B).

\section{Cell uptake of sunitinib}

The internalization of sunitinib in EPCs was assessed through immunofluorescence analysis and flow cytometry measurements, analyzing the cellular fluorescence intensity $\left(\lambda_{\text {exc }}=429 \mathrm{~nm}\right.$ and $\lambda_{\mathrm{em}}=540 \mathrm{~nm}$ ). The cell uptake of targeted liposomes was investigated using fluorescence confocal microscopy after exposure to free sunitinib $(10 \mu \mathrm{M})$, LNs-(sun) or RGD-LNs-(sun) containing $10 \mu \mathrm{M}$ of sunitinib. The treatment lasted $3 \mathrm{~h}$, to allow a significant cellular internalization without compromising cell viability. The nuclei were stained with DAPI. Cells exposed to RGD-LNs-(sun) showed an intense and diffuse fluorescence, higher than the one observed for cells incubated with free sunitinib or with LNs-(sun). 

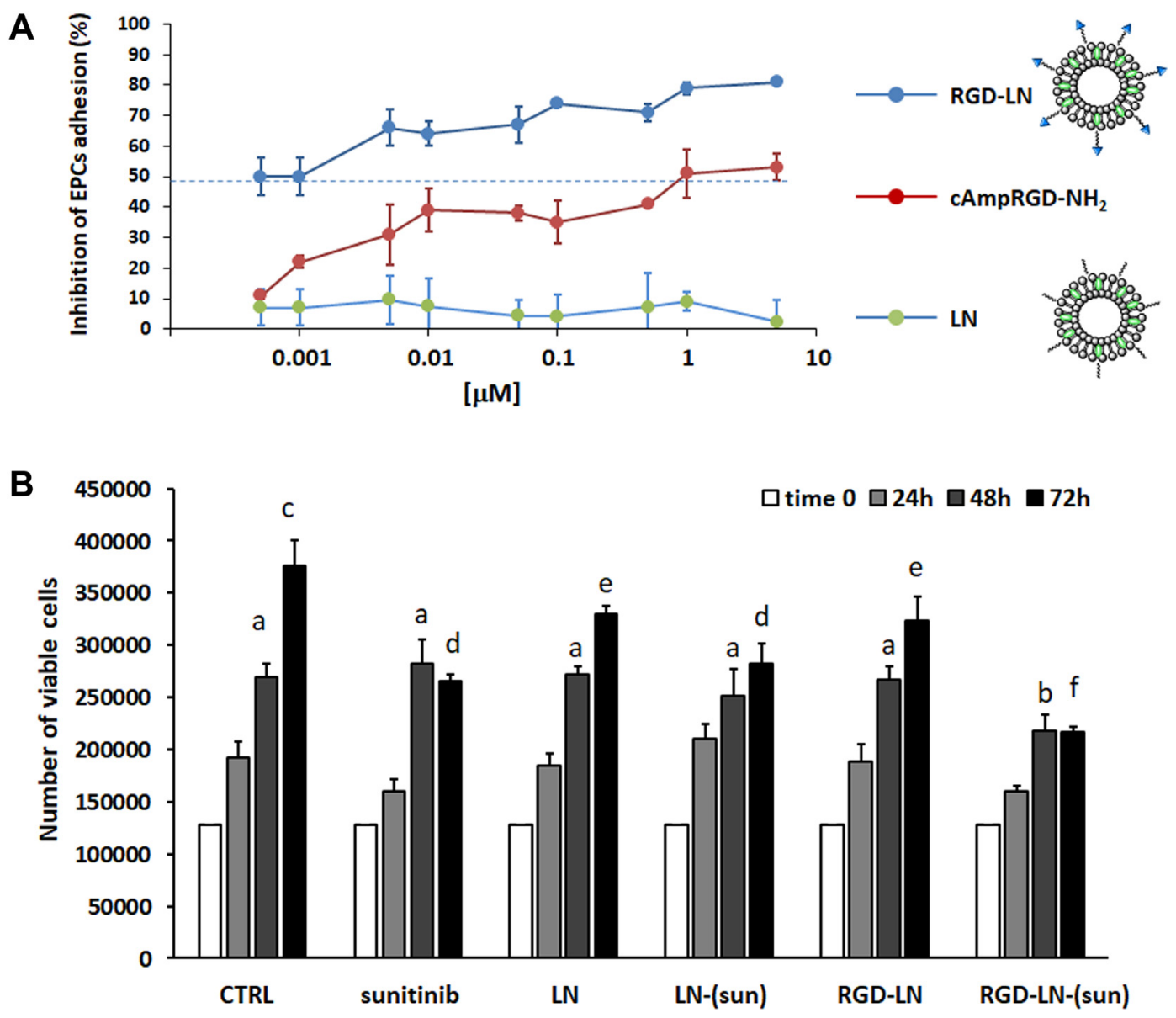

Figure 4. (A) Inhibition of EPCs adhesion to vitronectin expressed as mean value \pm SD of at least three independent experiments. (B) Inhibition of EPCs growth after treatment with sunitinib $1 \mu \mathrm{M}$, or different liposome formulations. Histograms refer to mean $\pm \mathrm{SD}(n=3)$ of the number of viable cells after $24 \mathrm{~h}, 48 \mathrm{~h}$, 72 h of treatment. Unlike letters (a, b, c, d, e, f) indicate significantly different means $P<0.05$ (see Statistical Analysis).

Interestingly, despite the brief incubation time, sunitinib accumulated in intracellular vesicles only when given as free drug (Figure 5, A). To further confirm the more efficient intracellular uptake of RGD-LNs-(sun) compared to sunitinib or LNs-(sun), a prolonged treatment at $1 \mu \mathrm{M}$ concentration was performed and evaluated using cytofluorimetric assay (Figure 5, B). The percentages of cellular populations showing a fluorescence intensity higher than the threshold value (cellular autofluorescence) are reported. Higher percentages mean higher intracellular sunitinib concentration (blank liposomes do not impact the overall cell autofluorescence, Figure S8). The data show that the targeted liposomes facilitate the uptake of sunitinib in comparison to both the untargeted liposomes and to the free drug. The gain in the sunitinib uptake by RGD-LNs-(sun) is more marked at shorter times ( $24 \mathrm{~h}$ and $48 \mathrm{~h}$ ), when a noticeably amount of fluorescent cells was measured. Instead, after $72 \mathrm{~h}$ exposition, the percentages of fluorescent cells for the two liposomes were similar but still higher than that of free sunitinib.

\section{Effect of RGD-liposome formulations on VEGFR2 phosphorylation and in vitro tubulogenesis}

In order to evaluate whether the VEGFR2 inhibiting activity of sunitinib is maintained even when administered by a liposome formulation, or it is even risen because of a concurrent inhibition of integrin $\alpha_{\mathrm{V}} \beta_{3}$, a VEGFR2 phosphorylation assay was performed. The ability of sunitinib $1 \mu \mathrm{M}$ as either free drug or in targeted RGD-LN-(sun) and untargeted LN-(sun) liposomes to inhibit VEGF-stimulated VEGFR2 phosphorylation was investigated by Western blotting using EPCs. Percent inhibition is reported in the densitometric analysis histogram (Figure 6, A). EPCs were treated for $24 \mathrm{~h}$ with the different compounds/ formulations and then activated with $50 \mathrm{ng} / \mathrm{mL}$ VEGF-A for $3 \mathrm{~min}$, before cell lysis for VEGFR2 phosphorylation detection. As expected, sunitinib strongly inhibits the VEGFR2 phosphorylation when administered both as a free drug and in liposome formulations. The best inhibition effect was obtained with the targeted liposomes, RGD-LN-(sun), and this can be correlated with their superior ability to be internalized. LNs-(sun) strongly inhibit VEGFR2 phosphorylation, but less efficiently than RGDLNs-(sun), in a manner comparable to that of free sunitinib. This evidence is still in line with the amount of sunitinib cell uptake measured by cytofluorimetric analysis.

To further investigate the anti-angiogenic effect of the sunitinib-loaded liposomes, we evaluated the in vitro tube formation of EPCs seeded on Matrigel (Corning). Cells were exposed to a medium containing VEGF-A $(20 \mathrm{ng} / \mathrm{mL})$ and 
A
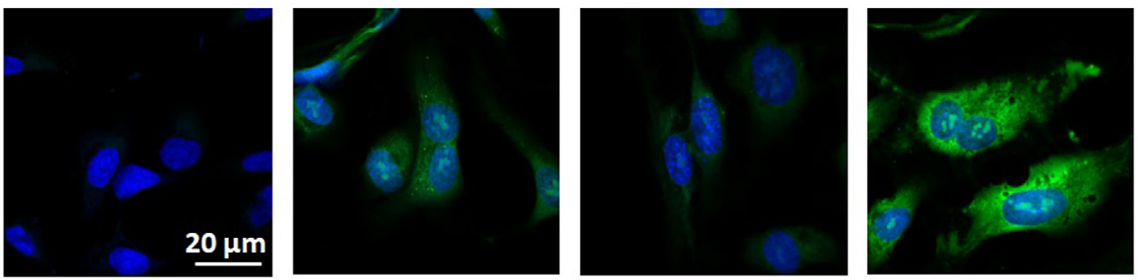

B

CTRL

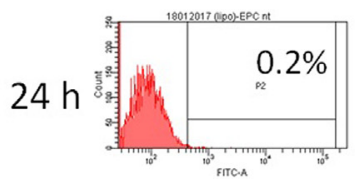

sunitinib

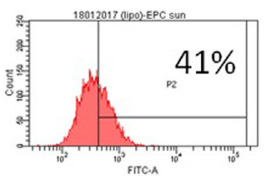

LN-(sun)

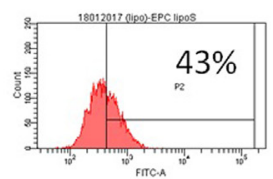

RGD-LN-(sun)

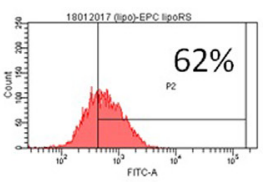

48
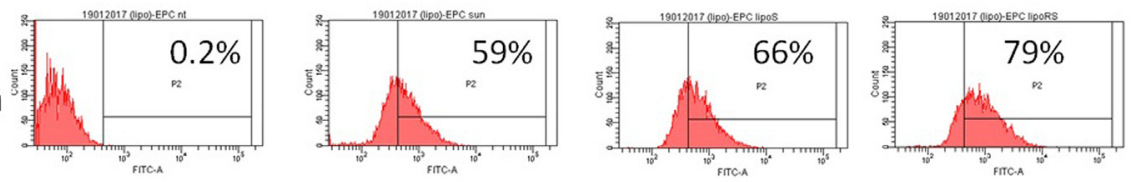

$72 \mathrm{~h}$
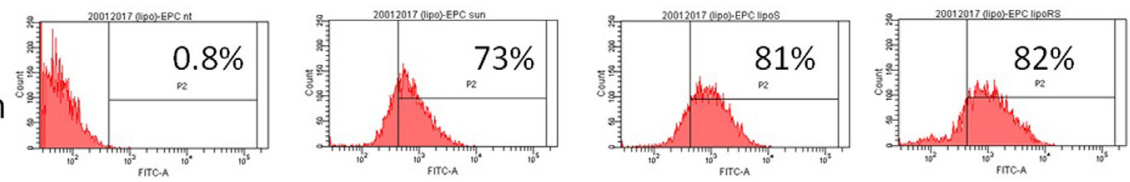

Figure 5. Immunofluorescence assay of EPCs treated for $3 \mathrm{~h}$ with free sunitinib $10 \mu \mathrm{M}, \mathrm{LN}$-(sun) or RGD-LN-(sun) at $10 \mu \mathrm{M}$ (A) and cytofluorimetric assay of EPCs treated daily for up to $72 \mathrm{~h}$ (B) with free sunitinib $1 \mu \mathrm{M}$, LN-(sun) or RGD-LN-(sun) at $1 \mu \mathrm{M}$. Representative images of at least three independent experiments.

incubated for $18 \mathrm{~h}$ in the presence of RGD-LN-(sun) or LN-(sun) at $1 \mu \mathrm{M}$ concentration of sunitinib. The anti-angiogenic activity was compared to that of free sunitinib $1 \mu \mathrm{M}, \mathrm{cAmpRGD}-\mathrm{NH}_{2}$ at $0.15 \mu \mathrm{M}$, and to the co-administration of sunitinib $(1 \mu \mathrm{M})$ and cAmpRGD- $\mathrm{NH}_{2}(0.15 \mu \mathrm{M})$. The choice of using cAmpRGD-NH $\mathrm{N}_{2}$ at $0.15 \mu \mathrm{M}$ is due to the fixed ratio [sunitinib]/[RGD] in the liposome formulation, that is $1 / 0.15$, hence the necessity of using the same concentration of the active ingredients (RGD or sunitinib) to compare their biological effects either as free drugs or within the liposome formulations. The antiangiogenic effect was reported as inhibition of capillary network formation (Figure 6, B, upper panel), given by the lower number of loops formed by connecting capillary projections (branches) with respect to the control experiment (Figure 6, $B$, lower panel).

The best in vitro inhibition of the capillary formation was given by the RGD-LNs-(sun) that, when administered at $1 \mu \mathrm{M}$ sunitinib concentration, were able to induce a $70 \%$ inhibition. In this experiment, the different activity of targeted and untargeted liposomes is evident. Interestingly, sunitinib as a free drug is markedly less effective with respect to the liposomal formulations, and this result can be ascribed to a lower cell uptake (Figure 5). cAmpRGD-NH $\mathrm{N}_{2}$ at low concentration $(0.15 \mu \mathrm{M})$ has a very slight antiangiogenic activity. Finally, a combined treatment of free sunitinib and cAmpRGD- $\mathrm{NH}_{2}$ was examined and the synergy of the two active units in the antiangiogenic activity was confirmed by an inhibition percentage higher than the sum of the single treatments.

\section{In vivo inhibition of angiogenesis}

Since the targeting effect of the RGD cannot be appreciated using in vitro assays, where the drug is added into the wells in direct contact with the cells, the in vivo anti-angiogenesis evaluation was performed, using a mice-implanted Matrigel plug assay. The ability of targeted RGD-LNs-(sun) to block angiogenesis was compared to that of the untargeted liposomes LNs-(sun) and sunitinib alone (as a malate salt). Matrigel sponges were subcutaneously injected into the flanks of nude mice, and compounds were daily intraperitoneally administered, with the aim to better appreciate the targeting effect of the RGD units. Plugs were removed from mice after 4 days of treatments and photographed. Despite the preliminary character of the data, a qualitative picture of the activity of the compounds was obtained (Figure 7). While the sponges recovered by untreated mice are crossed by a clear and evident blood vessel network, the sponges from treated mice show the effect of the antiangiogenic 


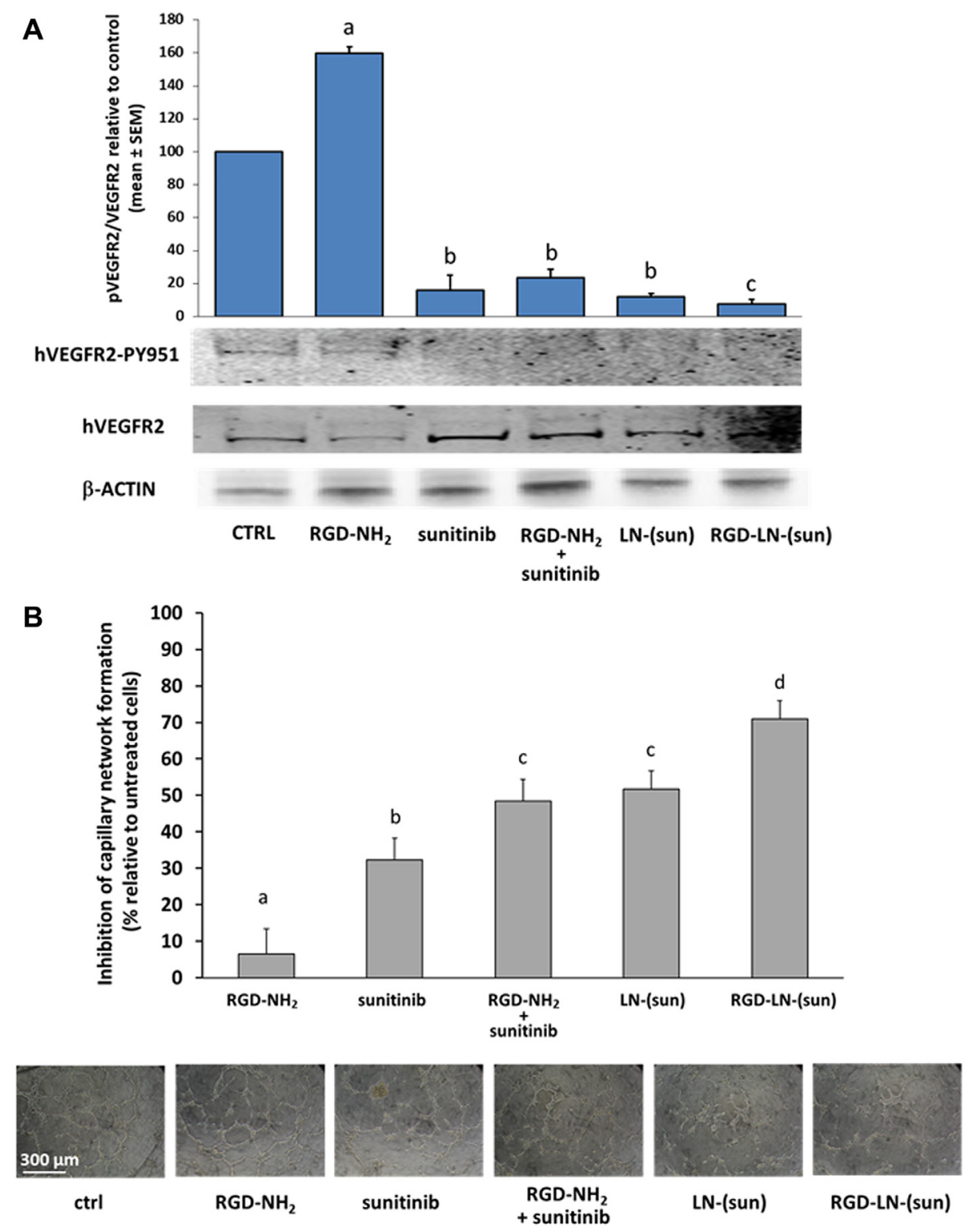

Figure 6. (A) Inhibition of VEGFR2 phosphorylation in EPCs treated with $1 \mu \mathrm{M}$ concentration of sunitinib, or different liposome formulations. Data are expressed as densitometric analysis compared to untreated cells and normalized to $\beta$-actin expression of three independent experiments (upper panel). Representative immunoblots (lower panel). (B) Inhibition of in vitro tubulogenesis of EPCs incubated for $18 \mathrm{~h}$ with $\mathrm{cAmpRGD-NH_{2 }}(0.15 \mu \mathrm{M})$, sunitinib $(1 \mu \mathrm{M}), \mathrm{cAmpRGD}-\mathrm{NH}_{2}(0.15 \mu \mathrm{M})+$ sunitinib $(1 \mu \mathrm{M}), \mathrm{LN}$-(sun) $(1 \mu \mathrm{M}$ referred to sunitinib) and RGD-LN-(sun) $(1 \mu \mathrm{M}$ referred to sunitinib). Histograms refer to mean \pm SD (three independent experiments) of the inhibition of branches development in three different fields as compared to untreated cells and expressed as percentage (upper panel). Representative images of the different treatments (lower panel). Unlike letters (a, b, c, d, e, f) indicate significantly different means $\mathrm{P}<0.05$ (see Statistical Analysis).

activity of sunitinib. When free sunitinib and LNs-(sun) were administered, only some tiny capillaries formed. Targeted liposomes RGD-LNs-(sun) gave an even better inhibition, in fact capillaries are pretty much absent in the plug. Noteworthy, the Matrigel plugs of mice treated with liposome formulations exhibit an intense yellow coloring, meaning high accumulation of sunitinib. This is observed also with the untargeted liposomes, suggesting that the liposome formulation itself could favor the accumulation in the Matrigel plug, when the leaky capillary network is insufficient to support drug clearance.

\section{Discussion}

The goal of this work was to develop targeted liposomes loaded with sunitinib for a potentiated inhibition of tumor angiogenesis by a spatio-temporal co-administration of sunitinib and an $\alpha_{\mathrm{V}} \beta_{3}$ antagonist. The cAmpRGD targeting units on the liposome surface should exert three main functions: i) to actively deliver the nanoparticle to the tumor site, ii) to increase the receptor-mediated cell uptake of liposomes and iii) to interfere in the $\alpha_{\mathrm{V}} \beta_{3}$-VEGFR2 cross-talk of angiogenic signal. Most of the literature examples of 

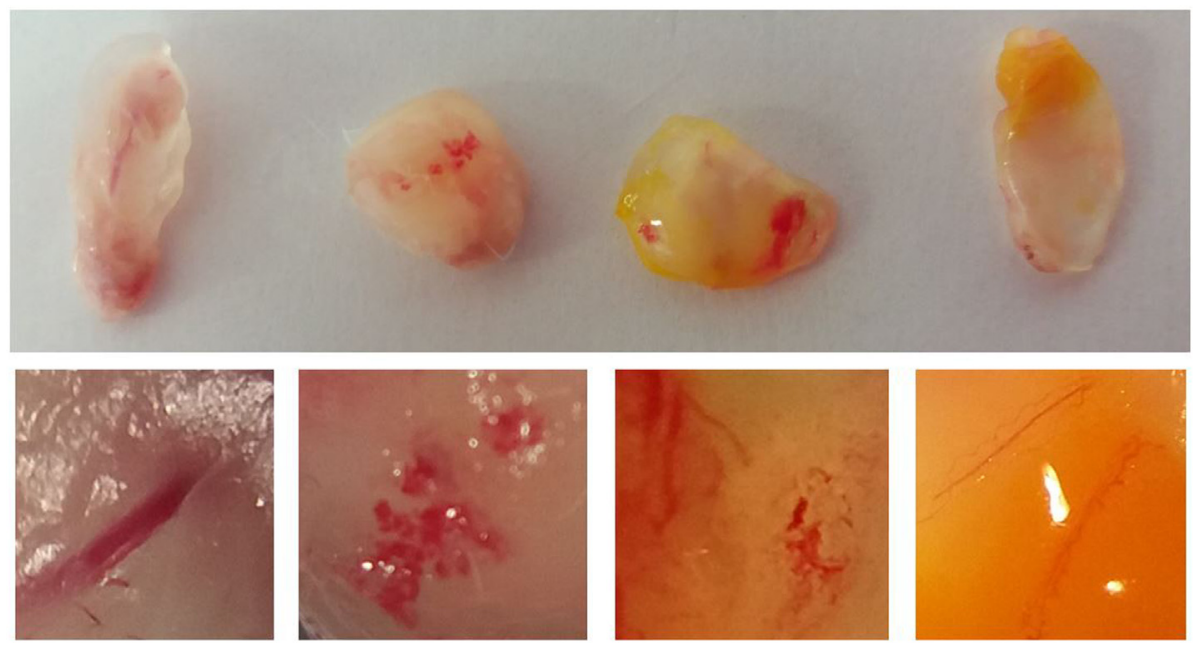

Figure 7. Inhibition of in vivo angiogenesis in Matrigel plugs implanted in FVB mice. Matrigel plugs contained VEGF-A/heparin. A daily IP administration of PBS (ctrl), $10 \mathrm{mg} / \mathrm{kg}$ of sunitinib or equivalent quantity within LN-(sun) or RGD-LN-(sun) was carried out. Plugs were removed from mice and photographed after 4 days (in the second line magnifications of the plugs above are reported).

RGD-liposomes proposed as antiangiogenic tools ${ }^{41}$ target a cytotoxic drug, such as doxorubicin or paclitaxel, to damage activated endothelial cells, ${ }^{42,43}$ while the attempts to target a TKI for an angiogenic signal modulation are rare. ${ }^{44}$ The few examples of sunitinib liposomes were developed in combination with a cytotoxic drug to combine the antitumor and antiangiogenic activity; sunitinib was co-loaded with irinotecan in the same untargeted liposome, ${ }^{45}$ or, more recently, sunitinib-loaded liposomes and vironelbine liposomes were co-administered to treat invasive breast cancer. ${ }^{46}$ To best of our knowledge, however, this is the first example of targeted RGD-liposomes loaded with sunitinib.

The plasma stability of the targeting unit cAmpRGD, a prerequisite for in vivo applications, was previously investigated ${ }^{27,28}$; its structural integrity was completely preserved under treatment in either human or rat plasma for 6-8 h. The DSPE-PEG-RGD 3 was used in the formulation of the targeted liposomes at a concentration of $5 \% \mathrm{~mol} / \mathrm{mol}$ with respect to the total amount of phospholipids. The lipid bilayer was formed by POPC $(55 \% \mathrm{~mol} / \mathrm{mol})$ and cholesterol (40\% $\mathrm{mol} / \mathrm{mol})$. POPC was chosen being probably the most diffused lipid in eukaryotic cells, while CHOL was added to rigidify the membrane and minimize the sunitinib leakage. The internal core was acidic thanks to $300 \mathrm{mM}$ concentration of $\left(\mathrm{NH}_{4}\right)_{2} \mathrm{SO}_{4}$ used as trapping agent. It has to be highlighted that both a rigid membrane and $\left(\mathrm{NH}_{4}\right)_{2} \mathrm{SO}_{4}$-enriched liposome interior were necessary for a stable encapsulation of sunitinib.

The liposomal formulation developed in this work presents suitable physicochemical characteristics for in vivo application. The average $\mathrm{R}_{\mathrm{H}}$ of $\sim 70 \mathrm{~nm}$ has been reported to be suitable for a preferred tumor accumulation via the EPR effect, with the consequence of a reduced uptake of sunitinib in healthy organs. The 5\% of PEG2000 lipid derivatives ensures longer circulation time by preventing opsonization through the induction of a fixed aqueous layer on the liposome surface. The length of $\mathrm{PEG}_{2000}$ chain, present in the formulation at percentages in the range 5$9.6 \% \mathrm{~mol} / \mathrm{mol}$, was reported to be optimal to ensure in vivo stealth properties of targeted liposomes. ${ }^{47}$
The purposely designed liposomes, through the optimized loading protocol, were able to encapsulate more than $95 \%$ of sunitinib present in the loading medium. Once encapsulated in the liposome inner aqueous pool, sunitinib is hardly released, as demonstrated by the leakage experiments, so that liposomes can be thought to safely carry the drug in the treated organism.

For the in vitro studies, the bone marrow-derived endothelial progenitor cells (EPCs) were used. In fact, EPCs are involved in tumor angiogenesis ${ }^{48,49}$ and it was reported that the levels of circulating EPCs are correlated with the malignancy of certain tumors in human patients. ${ }^{50,51}$ These cells overexpress the $\alpha_{V} \beta_{3}$ integrins and they upregulate the VEGFR2 expression when exposed to VEGF. ${ }^{52}$

The binding inhibition of EPCs to vitronectin was measured to assess the interaction between the RGD-liposomes and $\alpha_{\mathrm{V}} \beta_{3}$ receptors. The assay showed a strong adhesion inhibition of EPCs to vitronectin by the targeted liposomes (Figure 4, $A$ ), with an $\mathrm{IC}_{50}$ in the order of $1 \mathrm{nM}, 1000$ times lower than the $\mathrm{IC}_{50}$ measured with the reference monomer compound cAmpRGD$\mathrm{NH}_{2}$, revealing a clear multivalent effect of the multimeric presentation of RGD motifs on RGD-LNs. Conversely, the untargeted liposomes LNs did not show any ability to interact with the $\alpha_{V} \beta_{3}$ integrin, confirming that the adhesion inhibition registered with RGD-LNs was acted only by the RGD units and not due to the liposome structure.

In order to evaluate whether higher integrin binding caused higher internalization, the cell fluorescence was measured by flow cytometry and correlated to the intracellular sunitinib concentration. Free sunitinib can easily pass through the cell membrane and accumulate in the cytoplasm. ${ }^{28}$ After $24 \mathrm{~h}$, however, the amount of sunitinib internalized in EPCs was significantly higher when administered loaded in targeted RGDLNs. Targeted liposomes facilitate the sunitinib uptake with respect to the untargeted liposomes as well, likely via a receptormediated endocytosis. This conclusion was also supported by the cell growth inhibition experiments (Figure 4, B). In fact the blank liposomes LNs and RGD-LNs did not interfere in the cell 
growth, demonstrating the total safety of the nanocarriers proposed in this study, while the targeted RGD-LNs-(sun) had an inhibitory effect markedly higher than both the untargeted liposome LNs-(sun) and free sunitinib, results in line with the increased cell uptake of sunitinib.

Sunitinib loaded in liposomes is still able to work as a TKI inhibitor, meaning that it can escape the endosomes through which the RGD-LNs are presumably internalized and go to inhibit the VEGFR2 phosphorylation (Figure 6, A). Again, RGD-LNs-(sun) revealed to be the best formulation to maximize the sunitinib activity. Surprisingly, the cAmpRGD-NH $\mathrm{N}_{2}$ increased the VEGFR2 phosphorylation. We can postulate that the binding of the RGD-antagonist to $\alpha_{V} \beta_{3}$ integrin, at this concentration, causes a rapid phosphorylation of the VEGFR2 as compensatory mechanism.

To further investigate the effect of the targeted RGD-LNs(sun) on angiogenesis we evaluated, in vitro, the ability of EPCs to develop capillary network on a Matrigel-coated substratum and, in vivo, the ability of mice-resident endothelial cells to produce vessels in Matrigel plug assay. Tube-forming assays, in fact, offer an excellent overview of the molecular processes in angiogenesis, and can furnish an indication of the behavior of the inhibitors on the dynamic process of formation of new blood vessels. The in vitro assay showed that both LNs-(sun) and RGD-LNs-(sun) were able to efficiently inhibit the VEGF-A activated angiogenesis even though with a different efficiency (Figure $6, B$ ), and both were sensibly more active than free sunitinib ( $70 \%$ and $52 \%$ inhibition vs $32 \%)$. The superiority of RGD-LNs-(sun) with respect to LNs-(sun) can also be due to a contribution of RGD units in $\alpha_{\mathrm{V}} \beta_{3} / \mathrm{VEGFR} 2$ cross-talk inhibition. Lastly, the RGD-LNs-(sun) were definitely more efficient than the untargeted LNs-(sun) also in in vivo experiment, being able to inhibit completely the formation of new blood vessels. It is to be noted that the in vivo treatments were carried out at a suboptimal dose of sunitinib, showing that the targeted liposomes can allow the concentration of the administered drug to be decreased.

Thus, the RGD-decorated liposomes demonstrated to be a selective agent for antiangiogenic treatment. The combined action of cAmpRGD and sunitinib in the inhibition of $\alpha_{\mathrm{V}} \beta_{3}$ integrin and VEGFR2 and their cross-talk can be plausibly invocated, even if not unequivocally demonstrated. The key roles of the multiple cAmpRGD units in both targeting sunitinib-loaded liposomes and favoring their cell internalization are evident.

\section{Appendix A. Supplementary data}

Supplementary data to this article can be found online at https://doi.org/10.1016/j.nano.2019.02.015.

\section{References}

1. Weis SM, Cheresh DA. Tumor angiogenesis: molecular pathways and therapeutic targets. Nat Med 2011;17(11):1359-70.

2. Folkman J. Angiogenesis: an organizing principle for drug discovery? Nat Rev Drug Discov 2007;6(4):273-86.
3. Jain RK. Antiangiogenesis strategies revisited: from starving tumors to alleviating hypoxia. Cancer Cell 2014;26(5):605-22.

4. Ferrara N, Gerber H-P, LeCouter J. The biology of VEGF and its receptors. Nat Med 2003;9(6):669-76.

5. Hao Z, Sadek I. Sunitinib: the antiangiogenic effects and beyond. OncoTargets Ther 2016;9:5495-505.

6. Bergers G, Hanahan D. Modes of resistance to anti-angiogenic therapy. Nat Rev Cancer 2008;8(8):592-603.

7. Ye W. The complexity of translating anti-angiogenesis therapy from basic science to the clinic. Dev Cell 2016;37(2):114-25.

8. Gacche RN. Compensatory angiogenesis and tumor refractoriness. Oncogene 2015;4(6):e153.

9. Yang W-H, Xu J, Mu J-B, Xie J. Revision of the concept of antiangiogenesis and its applications in tumor treatment. Chronic Dis Transl Med 2017;3(1):33-40.

10. Cantelmo AR, Pircher A, Kalucka J, Carmeliet P. Vessel pruning or healing: endothelial metabolism as a novel target? Expert Opin Ther Targets 2017;21(3):239-47.

11. Wong PP, Bodrug N, Hodivala-Dilke KM. Exploring novel methods for modulating tumor blood vessels in cancer treatment. Curr Biol 2016;26 (21):R1161-6.

12. Barczyk M, Carracedo S, Gullberg D. Integrins. Cell Tissue Res 2010;339(1):269-80.

13. Seguin L, Desgrosellier JS, Weis SM, Cheresh DA. Integrins and cancer: regulators of cancer stemness, metastasis, and drug resistance. Trends Cell Biol 2015;25(4):234-40.

14. Avraamides CJ, Garmy-Susini B, Varner JA. Integrins in angiogenesis and lymphangiogenesis. Nat Rev Cancer 2008;8:604-17.

15. Atkinson SJ, Ellison TS, Steri V, Gould E, Robinson SD. Redefining the role(s) of endothelial $\alpha_{\mathrm{V}} \beta_{3}$-integrin in angiogenesis. Biochem Soc Trans 2014;42(6):1590-5.

16. Ivaska J, Heino J. Cooperation between integrins and growth factor receptors in signaling and endocytosis. Annu Rev Cell Dev Biol 2011;27 (1):291-320.

17. Reynolds AR, Reynolds LE, Nagel TE, Lively JC, Robinson SD, Hicklin DJ, et al. Elevated Flk1 (vascular endothelial growth factor receptor 2) signaling mediates enhanced angiogenesis in beta3-integrin-deficient mice. Cancer Res 2004;64:8643-50.

18. Somanath PR, Malinin NL, Byzova TV. Cooperation between integrin $\alpha_{V} \beta_{3}$ and VEGFR2 in angiogenesis. Angiogenesis 2009;12(2):177-85.

19. Wu J, Strawn TL, Luo M, Wang L, Li R, Ren M, et al. Plasminogen activator inhibitor-1 inhibits angiogenic signaling by uncoupling vascular endothelial growth factor receptor- $2-\alpha_{\mathrm{V}} \beta_{3}$ integrin cross talk. Arterioscler Thromb Vasc Biol 2015;35(1):111-20.

20. Marelli UK, Rechenmacher F, Sobahi TRA, Mas-Moruno C, Kessler H. Tumor targeting via integrin ligands. Front Oncol 2013;3:1-12.

21. Arosio D, Casagrande C. Advancement in integrin facilitated drug delivery. Adv Drug Deliv Rev 2016;97:111-43.

22. Raab-Westphal S, Marshall JF, Goodman SL. Integrins as therapeutic targets: successes and cancers. Cancers (Basel) 2017;9(9):1-28.

23. Stupp R, Hegi ME, Gorlia T, Erridge SC, Perry J, Hong YK, et al. Cilengitide combined with standard treatment for patients with newly diagnosed glioblastoma with methylated MGMT promoter (CENTRIC EORTC 26071-22072 study): a multicentre, randomised, open-label, phase 3 trial. Lancet Oncol 2014;15(10):1100-8.

24. Hamidi H, Pietilä M, Ivaska J. The complexity of integrins in cancer and new scopes for therapeutic targeting. Cancer 2016;115(9):1017-23.

25. Demircioglu F, Hodivala-Dilke K. $\alpha v \beta 3$ integrin and tumour blood vessels - learning from the past to shape the future. Curr Opin Cell Biol 2016;42:121-7.

26. Zanardi F, Burreddu P, Rassu G, Auzzas L, Battistini L, Curti C, et al. Discovery of subnanomolar arginine-glycine-aspartate-based $\alpha_{V} \beta_{3} / \alpha_{V} \beta_{5}$ integrin binders embedding 4-aminoproline residues. $\mathrm{J} \mathrm{Med} \mathrm{Chem}$ 2008;51(6):1771-82.

27. Sartori A, Bianchini F, Migliari S, Burreddu P, Curti C, Vacondio F, et al. Synthesis and preclinical evaluation of a novel, selective ${ }^{111} \mathrm{In}-$ 
labelled aminoproline-RGD-peptide for non-invasive melanoma tumor imaging. Med Chem Commun 2015;6(12):2175-83.

28. Sartori A, Portioli E, Battistini L, Calorini L, Pupi A, Vacondio F, et al. Synthesis of novel c(AmpRGD)-sunitinib dual conjugates as molecular tools targeting the $\alpha \mathrm{v} \beta 3$ integrin/VEGFR2 couple and impairing tumorassociated angiogenesis. J Med Chem 2017;60(1):248-62.

29. Battistini L, Burreddu P, Sartori A, Arosio D, Manzoni L, Paduano L, et al. Enhancement of the uptake and cytotoxic activity of doxorubicin in cancer cells by novel cRGD-semipeptide-anchoring liposomes. Mol Pharm 2014;11(7):2280-93.

30. Noble GT, Stefanick JF, Ashley JD, Kiziltepe T, Bilgicer B. Ligandtargeted liposome design: challenges and fundamental considerations. Trends Biotechnol 2014;32(1):32-45.

31. Danhier F, Le Breton A, Préat V. RGD-based strategies to target alpha(v) beta (3) integrin in cancer therapy and diagnosis. Mol Pharm 2012;9(11):2961-73.

32. Russo Krauss I, Imperatore R, De Santis A, Luchini A, Paduano L, D'Errico G. Structure and dynamics of cetyltrimethylammonium chloride-sodium dodecylsulfate (CTAC-SDS) catanionic vesicles: high-value nano-vehicles from low-cost surfactants. J Colloid Interface Sci 2017;501:112-22.

33. Haran G, Cohen R, Bar LK, Barenholz Y. Transmembrane ammonium sulfate gradients in liposomes produce efficient and stable entrapment of amphipathic weak bases. BBA-Biomembranes 1993;1151(2):201-15.

34. Siepmann J, Faisant N, Akiki J, Richard J, Benoit JP. Effect of the size of biodegradable microparticles on drug release: experiment and theory. $J$ Control Release 2004;96(1):123-34.

35. Fritze A, Hens F, Kimpfler A, Schubert R, Peschka-Süss R. Remote loading of doxorubicin into liposomes driven by a transmembrane phosphate gradient. Biochim Biophys Acta Biomembr 2006;1758 (10):1633-40.

36. Margheri F, Chillà A, Laurenzana A, Serratì S, Mazzanti B, Saccardi R, et al. Endothelial progenitor cell - dependent angiogenesis requires localization of the full-length form of uPAR in caveolae. Blood 2011;118(13):3743-55.

37. D'Errico G, Silipo A, Mangiapia G, Vitiello G, Radulescu A, Molinaro A, et al. Characterization of liposomes formed by lipopolysaccharides from Burkholderia cenocepacia, Burkholderia multivorans and Agrobacterium tumefaciens: from the molecular structure to the aggregate architecture. Phys Chem Chem Phys 2010;12(41):13574-85.

38. Vitiello G, Falanga A, Petruk AA, Merlino A, Fragneto G, Paduano L, et al. Fusion of raft-like lipid bilayers operated by a membranotropic domain of the HSV-type I glycoprotein gH occurs through a cholesteroldependent mechanism. Soft Matter 2015;11(15):3003-16.

39. De Santis A, La Manna S, Krauss IR, Malfitano AM, Novellino E, Federici L, et al. Nucleophosmin-1 regions associated with acute myeloid leukemia interact differently with lipid membranes. Biochim Biophys Acta Gen Subj 2018;1862(4):967-78.
40. Oliva R, Emendato A, Vitiello G, De Santis A, Grimaldi M, D'Ursi AM, et al. On the microscopic and mesoscopic perturbations of lipid bilayers upon interaction with the MPER domain of the HIV glycoprotein gp41. Biochim Biophys Acta Biomembr 2016;1858(8):1904-13.

41. Duro-Castano A, Gallon E, Decker C, Vicent MJ. Modulating angiogenesis with integrin-targeted nanomedicines. Adv Drug Deliv Rev 2017;119:101-19.

42. Murphy EA, Majeti BK, Barnes LA, Makale M, Weis SM, Lutu-Fuga K, et al. Nanoparticle-mediated drug delivery to tumor vasculature suppresses metastasis. S A 2008;105(27):9343-8.

43. Liu Y, Mei L, Yu Q, Xu C, Qiu Y, Yang Y, et al. Multifunctional tandem peptide modified paclitaxel-loaded liposomes for the treatment of vasculogenic mimicry and cancer stem cells in malignant glioma. ACS Appl Mater Interfaces 2015;7(30):16792-801.

44. Wang JL, Xi Y, Liu YL, Wang ZH, Zhang Q. Combination of targeted PDT and anti-VEGF therapy for rat CNV by RGD-modified liposomal photocyanine and sorafenib. Investig Ophthalmol Vis Sci 2013;54 (13):7983-9.

45. Maitani Y, Saito H, Seishi Y, Iwase Y, Yamauchi T, Higashiyama K, et al. A combination of liposomal sunitinib plus liposomal irinotecan and liposome co-loaded with two drugs enhanced antitumor activity in PC12-bearing mouse. J Drug Target 2012;20(10):873-82.

46. Shi JF, Sun MG, Li XY, Zhao Y, Ju RJ, Mu LM, et al. A combination of targeted sunitinib liposomes and targeted vinorelbine liposomes for treating invasive breast cancer. J Biomed Nanotechnol 2015;11 (9):1568-82.

47. Lee CM, Choi Y, Huh EJ, Lee KY, Song HC, Sun MJ, et al. Polyethylene glycol (PEG) modified 99mTc-HMPAO-liposome for improving blood circulation and biodistribution: the effect of the extent of PEGylation. Cancer Biother Radiopharm 2005;20(6):620-8.

48. Gao D, Nolan DJ, Mellick AS, Bambino K, McDonnell K, Mittal V. Endothelial progenitor cells control the Angiogenic switch in mouse lung metastasis. Science 2008;319(5860):195-8.

49. Plummer PN, Freeman R, Taft RJ, Vider J, Sax M, Umer BA, et al. MicroRNAs regulate tumor angiogenesis modulated by endothelial progenitor cells. Cancer Res 2013;73(1):341-52.

50. Dome B, Timar J, Dobos J, Meszaros L, Raso E, Paku S, et al. Identification and clinical significance of circulating endothelial progenitor cells in human non-small cell lung cancer. Cancer Res 2006;66(14):7341-7.

51. Naik RP, Jin D, Chuang E, Gold EG, Tousimis EA, Moore AL, et al. Circulating endothelial progenitor cells correlate to stage in patients with invasive breast cancer. Breast Cancer Res Treat 2008;107 (1):133-8.

52. Bagley RG, Rouleau C, St. Martin T, Boutin P, Weber W, Ruzek M, et al. Human endothelial precursor cells express tumor endothelial marker 1/ endosialin/CD248. Mol Cancer Ther 2008;7(8):2536-46. 